\title{
BEHAVIOUR OF GREEN LINES AT ROYDEN'S BOUNDARY OF RIEMANN SURFACES
}

\author{
MITSURU NAKAI
}

To Professor Kinjiro Kunugi on the occasion of his 60th birthday

The aim of this paper is to investigate the behaviour of Green lines at Royden's boundary $\Gamma$ of a Riemann surface $R$ with the Green function $g(z, o)$ with the fixed pole $o$ in $R$. We denote by $\&$ the totality of Green lines $L$ issuing from the fixed point $o$. There exists a positive number $\varepsilon$ such that the set $(z \in R ; g(z, o)>-\log \varepsilon)$ is relatively compact and simply connected in $R$ and the set $\mathbf{J}=(z \in R ; g(z, o)=-\log \varepsilon)$ is homeomorphic ${ }^{*)}$ to the unit circle. We may represent each point $z$ in $\mathbf{J}$ by $\theta \quad(0 \leq \theta<2 \pi)$, if $z$ corresponds to $e^{i \theta}$ by the above homeomorphism. Using this, we can represent each $L$ in $\&$ by $L=L_{\theta}(0 \leq \theta<2 \pi)$, where $\theta$ is the point determined as the intersection of $L$ and J. For each set $S$ in $\Gamma$, we set $\widetilde{S}=\left(\theta ;\left(\check{L}_{\theta} \cap \Gamma\right) \cap S \neq \varnothing\right)$ and $\check{S}=\left(\theta ;\left(\bar{L}_{\theta} \cap \Gamma\right) \subset S\right)$. We denote by $\bar{m}$ (resp. $\underline{m}$ ) the outer (resp. inner) normalized Lebesgue measure on J. These may be considered as the outer and inner measures on $\mathbb{B}$. For a measurable set, we set $m=\bar{m}$. We also denote by $\mu$ the canonical measure on $\Gamma$ with the center $o$ (i.e. the harmonic measure for subsets of $I$ calculated at $o$ ).

The fundamental result of this paper is that

$$
\bar{m}(\widetilde{K}) \leq \mu(K) \quad \text { for any } F_{\sigma} \text { set } K \text { in } \Gamma,
$$

or equivalently that

$$
\underline{m}(\check{U}) \geq \mu(U) \quad \text { for any } G_{\delta} \text { set } U \text { in } \Gamma .
$$

Since the subset of $\Gamma$ at each point of which the Green function is strictly positive is an $F_{o}$ set with canonical measure zero, the first inequality mentioned above implies the well-known Brelot-Choquet's result [2] that the set of Green lines on which the Green function does not tend to zero is of Lebesgue measure zero.

By using the above inequalities and the theory of Royden's compactification,

Received May 10, 1963.

*) This homeomorphism is a special one. See $\$ 2$. 
we shall investigate the behaviour of $H D$ and $A D$ functions on Green lines. By a simple application of Riesz-Fisher's theorem, it is seen that the "radial limit"

$$
f(\theta)=\lim _{L_{\theta} \ni z \rightarrow \Gamma} f(z)
$$

exists for every $\theta$ in $[0,2 \pi]$ except a set of measure zero, where $f$ is an arbitrary a.c.T. function on $R$ with finite Dirichlet integral taken over $R$. This also follows from a result of Godefroid [3]. Hence, in particular, any $H D$ function $\boldsymbol{u}$ on $R$ possesses the radial limit $u(\theta)$ almost everywhere on $\mathbf{J}$. Concerning this, we shall show an analogue of the mean-value theorem of Gauss:

$$
u(o)=\frac{1}{2 \pi} \int_{0}^{2 \pi} u(\theta) d \theta
$$

for any $u$ in $H D(R)$. We shall also show that

$$
(u \wedge v)(\theta)=u(\theta) \cap v(\theta)
$$

for any $u$ and $v$ in $H D(R)$, where $u \wedge v$ is the greatest harmonic minorant of $u$ and $v$ and $a \cap b=\min (a, b)$. Similarly, any $A D$-function $f$ on $R$ possesses the radial limit $f(\theta)$ almost everywhere on $\mathbf{J}$. Concerning this, we shall show an analogoue of $\mathrm{F}$. and M. Riesz's theorem: if $f$ is an $A D$-function on $R$ such that $f(\theta)=0$ on a subset of $\mathbf{J}$ with positive measure, then $f$ vanishes identically on $R$. This follows also from a result of Brelot-Choquet [2]. Our main result in this paper is as follows.

$A$ hyperbolic Riemann surface $R$ belongs to the Constantinescu-Cornea's class $U_{H D}$ if and only if there exists a subset $P$ of $\mathbf{J}$ with positive measure such that $u(\theta)$ is a constant almost everywhere on $P$ for any HD-function $u$ on $R$ (Theorem 3$)$.

\section{Green lines and polar coordinate}

1. Let $R$ be a hyperbolic Riemann surface and $o$ be a fixed point in $R$ and $g(z, o)$ be the Green function on $R$ with its pole $o$. Consider a pair $(r(z), \theta(z))$ of functions of local parameters $z$ defined by the following equations:

$$
\left\{\begin{array}{l}
d r(z) / r(z)=-d g(z, o) \\
d \theta(z)=-* d g(z, o)
\end{array}\right.
$$


The function $r(z)$ is uniquely determined by the initial condition $r(o)=0$ as a single-valued function on $R$, i.e.

$$
r(z)=\exp (-g(z, o)) .
$$

Clearly $0 \leq r(z)<1$ on $R$. We set

$$
G_{\mathrm{p}}=(z \in R ; r(z)<\rho) \text { and } C_{p}=\partial G_{p}
$$

for each number $\rho$ in $0<\rho<1$. The set $C_{\rho}$ is nothing but the $\log (1 / \rho)$-level curve for $g(z, o)$. We say that $C_{\rho}$ (resp. $G_{p}$ ) is regular if $d g(z, o) \neq 0$ on $C_{\rho}$. Concerning the set $G_{p}$, the following is very important.

Lemma 1 (Kuramochi's lemma). Assume that the set $G_{p}$ is regular. Then the set $G_{p}$ is a subdomain of $R$ and the double $\hat{G}_{\rho}$ of $G_{\rho}$ along the relative bounda$r y C_{p}$ is a Riemann surface with null boundary.

For the proof of this, see Kusunoki-Mori [4] or Nakai [7]. Using this, we prove

LEMMA 2. For any regular $C_{\rho}, \frac{1}{2 \pi} \int_{C p} d \theta(z)=1$.

Proof. Let $\left(R_{n}\right)_{0}^{\infty}$ be a normal exhaustion of $R$ with $o \in R_{0} \subset \bar{R}_{0} \subset G_{p}$ and $w_{n}(z)$ be the harmonic function on $G_{p} \cap R_{n}-R_{0}$ with the continuous boundary value 1 on $\partial R_{0}$ and 0 on $\partial R_{n} \cap G_{p}$ and the normal derivative $\partial w_{n} / \partial \nu=0$ on $\partial G_{p} \cap R_{n}$. For convinience, we set $w_{n}=0$ outside $R_{n}$ in $G_{\rho}$ and $w_{n}=1$ on $R_{0}$. Then by Lemma $1, w_{n} \nearrow 1$ on $\bar{G}_{p}$ and $D_{G_{p}}\left(w_{n}\right) \searrow 0$. By Green's formula,

$$
D_{G_{p}}\left(w_{n, g}\right)=\int_{C_{\rho} \cap R_{n}} w_{n}(z) \frac{\partial}{\partial \nu} g(z, o) d s+\int_{\partial R_{0}} \frac{\partial}{\partial \nu} g(z, o) d s,
$$

where $\frac{\partial}{\partial \nu}$ denotes the inner normal differentiation with respect to the open set $G_{p} \cap R_{n}-\bar{R}_{0}$ and $d s$ denotes the line element on $C_{\rho}$. Here

$$
\int_{\partial R_{0}} \frac{\partial}{\partial \nu} g(z, o) d s=-2 \pi
$$

and by Lebesgue's convergence theorem,

$$
\lim _{n} \int_{C_{p} \cap R_{n}} w_{n}(z) \frac{\partial}{\partial \nu} g(z, o) d s=\int_{c_{p}} \frac{\partial}{\partial \nu} g(z, o) d s=\int_{C_{p}} d \theta(z) .
$$

On the other hand,

$$
D_{G_{p}}\left(w_{n}, g\right) \leq \sqrt{D_{G_{p}-R_{0}}(g) D_{G_{p}}\left(w_{n}\right)} \searrow 0
$$


as $n \nearrow \infty$. Hence we get $\int_{c_{p}} d \theta(z)=2 \pi$.

Q.E.D.

2. Although $\theta(z)$ is not single-valued in $R-(o)$, it is harmonic locally on $R-(o)$. A level arc for $\theta(z)$ is an open or closed or half open and closed arc on which $d \theta \neq 0$ and $\theta(z)$ is a constant, being locally considered at each point. We call a level arc for $\theta(z)$ as a Green arc. The totallity of Green arcs forms a partially ordered set by inclusion. In this sense, a maximal Green arc is called a Green line. Hereafter we use the term Green line $L$ only for those $L$ issuing from $o$, i.e. $L \ni o$. We denote by

(83

the totality of Green lines (issuing from $o$ ) and we call $\mathbb{B}$ as the space of Green lines. We denote by

$$
R^{g}
$$

the set of all points in $R$ which lie on a Green line issuing from $o$. Clearly $R^{g}$ is a subdomain of $R$. If we choose $\varepsilon$ sufficiently small, then the set $G_{\varepsilon}$ is regular and relatively compact in $R$ and conformally equivalent to the disc $(z ;|z|<1)$. Hereafter we fix such an $\varepsilon$ and use the following particular notations :

$$
\xi=G_{\varepsilon}=(z \in R ; r(z)<\varepsilon)
$$

and

$$
\mathbf{J}=\partial \xi=C_{\varepsilon}=(z \in R ; r(z)=\varepsilon) \text {. }
$$

Since there exists a one-to-one analytic mapping $\varphi$ of the $\operatorname{disc}(z ;|z| \leq 1)$ onto the set $\& \cup \mathbf{J}$, we can represent each point $p$ in $\mathbf{J}$ by the coordinate $\theta$ in $[0,2 \pi)$, where the correspondence $p \leftrightarrow \theta$ is given by the relation $\varphi\left(e^{i \theta}\right)=p$. Using this, we can represent each Green line $L$ issuing from $o$ by

$$
L=L_{\theta},
$$

where $\theta$ is the point $L \cap \mathbf{J}$, or more precisely, $\theta$ is the coordinate of the point $L \cap \mathbf{J}$ in $\mathbf{J}$. Hence we can write

$$
\mathbb{B}=\left(L_{\theta} ; \theta \in \mathbf{J}\right) \text {. }
$$

Since the totality of points in $R$ at which $d \theta(z)=0$ is countable, the set 
$\mathbf{E}=\left(\theta ; L_{\theta} \in \mathbb{S}\right.$ and $d \theta(z)=0$ at the end point $L_{\theta}-L_{\theta} \cup(o)$ of $\bar{L}_{\theta}$ in $\left.R\right)$ is a countable subset of $\mathbf{J}$ and hence of Lebesgue measure zero.

3. Although $\theta(z)$ is not single-valued on $R$, we may use $r(z) e^{i \theta(z)}$ as a local parameter at each point of $R$ except possibly a countable number of points at which $d \theta(z)=0$. If we take the branch of $\theta(z)$ at $z_{0} \in L_{\theta}$ such as $\theta\left(z_{0}\right)=\theta$, then we can use the single-valued function

$$
r(z) e^{i \theta(z)}=r e^{i \theta}
$$

in $R^{g}$ as the global polar coordinate in $R^{g}$ with the origin $o$. We also denote by

\section{$m$}

the normalized Lebesgue measure on $\mathbf{J}$, i.e.

$$
d m(\theta)=\frac{1}{2 \pi} d \theta
$$

Using these concepts, we give a generalization of the mean-value theorem of Gauss on bounded harmonic functions. As usual, we denote by $H B=H B(R)$ the totality of bounded harmonic functions on $R$.

Proposition 1 (Gauss' theorem). Let $\rho$ be an arbitrary number in $0<\rho<1$ with regular $C_{\rho}$ and $u$ be in $H B\left(G_{\rho}\right)$ and continuous in $G_{p} \cup C_{\rho}$. Then

$$
u(o)=\int_{0}^{2 \pi} u\left(\rho e^{i \theta}\right) d m(\theta) .
$$

Proof. Let $\left(R_{n}\right)_{1}^{\infty}$ be a normal exhaustion of $R$ with $\bar{E} \subset R_{1}$ and $g_{n}(z, o)$ be the Green function on $G_{\rho} \cap R_{n}$ with its pole $o$. For convinience, we set $g_{n}(z, o)$ $=0$ outside $\bar{R}_{n}$ in $G_{p}$. Clearly $g(z, o)-\rho$ is the Green function on $G_{\rho}$ with the pole $o$. Hence $g_{n}(z, o) \nearrow g(z, o)-\rho$ on $G_{p}$. Therefore by defining $\frac{\partial}{\partial \nu} g_{n}(z, o)=0$ on $\mathrm{C}_{\rho}-C_{\rho} \cap R_{n}$, we have

$$
0 \leq \frac{\partial}{\partial \nu} g_{n}(z, o) \nearrow \frac{\partial}{\partial \nu} g(z, o)
$$

on $C_{\rho}$, where $\frac{\partial}{\partial \nu}$ denotes the inner normal differentiation with respect to $G_{p}$.

Next we denote by $w_{n}$ the harmonic functionon $G_{\rho} \cap R_{n}-\&$ with the continuous boundary value 0 on $J=\partial \&$ and 1 on $\partial R_{n} \cap G_{\rho}$ and $\frac{\partial}{\partial \nu} w_{n}=0$ on $\partial G_{\rho} \cap R_{n}$. 
For convinience, we set $w_{n}=0$ on $\left[\right.$ and $w_{n}=1$ on $G_{p}-R_{n}$. Then by Lemma 1 ,

$$
w_{n} \searrow 0 \text { on } \bar{G}_{\rho} \text { and } D_{\theta_{p}}\left(w_{n}\right) \searrow 0
$$

as $n \nearrow \infty$. By Green's formula,

$$
\begin{aligned}
0 & \leq \int_{\partial R_{n} \cap G_{\rho}} \frac{\partial}{\partial \nu} g_{n}(z, o) d s+\int_{C_{\rho} \cap R_{n}} w_{n}(z) \frac{\partial}{\partial \nu} g_{n}(z, o) d s \\
& =\int_{\partial\left(G_{p} \cap R_{n}-R_{0}\right)} w_{n}(z) \frac{\partial}{\partial \nu} g_{n}(z, o) d s \\
& \leq \sqrt{D_{G_{\rho}-\bar{E}}\left(g_{n}\right) \cdot D_{G_{\rho}}\left(w_{n}\right)} \leq \sqrt{D_{G_{p}-\bar{g}}(g) \cdot D_{G_{\rho}}\left(w_{n}\right)} \searrow 0
\end{aligned}
$$

as $n \nearrow_{\infty}$. On the other hand,

$$
0 \leq w_{n}(z) \frac{\partial}{\partial \nu} g_{n}(z, o) \leq \frac{\partial}{\partial \nu} g(z, o) \text { on } C_{\rho}
$$

and $w_{n}(z) \frac{\partial}{\partial \nu} g_{n}(z, o) \rightarrow 0$ on $C_{\mathrm{p}}$ and $\frac{\partial}{\partial \nu} g(z, o) d s$ is integrable on $C_{p}$. Hence by the Lebesgue convergence theorem,

$$
\lim _{n} \int_{C_{p} \cap R_{n}} w_{n}(z) \frac{\partial}{\partial \nu} g_{n}(z, o) d s=0 .
$$

Therefore, we get

$$
\lim _{n} \int_{\partial R_{n} \cap G_{p}} \frac{\partial}{\partial \nu} g_{n}(z, o) d s=0 .
$$

Again by Green's formula,

$$
\begin{aligned}
u(o) & =\frac{1}{2} \int_{\partial\left(G_{\rho} \cap R_{n}\right)} u(z) \frac{\partial}{\partial \nu} g_{n}(z, o) d s \\
& =\frac{1}{2 \pi} \int_{C_{\rho} \cap R_{n}} u(z) \frac{\partial}{\partial \nu} g(z, o) d s+\frac{1}{2 \pi} \int_{\partial R_{n} \cap \theta_{p}} u(z) \frac{\partial}{\partial \nu} g_{n}(z, o) d s .
\end{aligned}
$$

Here we have

$$
\left|\int_{\partial R_{n} \cap \theta_{\rho}} u(z) \frac{\partial}{\partial \nu} g_{n}(z, o) d s\right| \leq\left(\sup _{G_{p}}|u|\right) \cdot \int_{\partial R_{n} \cap \theta_{p}} \frac{\partial}{\partial \nu} g_{n}(z, o) d s \rightarrow 0
$$

as $n \nearrow \infty$. We also have

$$
\int_{C_{p} \cap R_{n}} u(z) \frac{\partial}{\partial \nu} g_{n}(z, o) d s \rightarrow \int_{C_{p}} u(z) \frac{\partial}{\partial \nu} g(z, o) d s .
$$

In fact,

$$
\left|u(z) \frac{\partial}{\partial \nu} g_{n}(z, o)\right| \leq\left(\sup _{G_{\rho}}|u|\right) \frac{\partial}{\partial \nu} g(z, o)
$$


and

$$
u(z) \frac{\partial}{\partial \nu} g_{n}(z, o) \rightarrow u(z) \frac{\partial}{\partial \nu} g(z, o)
$$

as $n \rightarrow \infty$ on $C_{p}$ and $\left(\sup _{a_{\rho}}|u|\right) \frac{\partial}{\partial \nu} g(z, o) d s$ is integrable on $C_{p}$. Hence by Lebesgue's convergence theorem, we get the above conclusion.

Thus by making $n \rightarrow \infty$, we get

$$
u(o)=\frac{1}{2 \pi} \int_{C_{\rho}} u(z) \frac{\partial}{\partial \nu} g(z, o) d s
$$

On the other hand,

$$
\frac{\partial}{\partial \nu} g(z, o) d s=d \theta(z)
$$

on $C_{\rho}$. Hence by using local parameter $z=r e^{i \theta}$, we finally get

$$
u(o)=\frac{1}{2 \pi} \int_{0}^{2 \pi} u\left(\rho e^{i \theta}\right) d \theta=\int_{0}^{2 \pi} u\left(\rho e^{i \theta}\right) d m(\theta) .
$$

Q.E.D.

\section{End parts of Green lines in Royden's boundary}

4. We denote by $M(R)$ the (real) Royden's algebra associated with the surface $R$, i.e. the algebra of all real-valued bounded a.c.T. (abbreviation of the term absolutely continuous in the sense of Tonelli") functions on $R$ with finite Dirichlet integrals taken over $R$ (see Nakai [5], [6]). We denote by $R^{*}$ the Royden compactification of $R$, i.e. the compact Hausdorff space containing $R$ as its open and dense subspace, and the algebra $M(R)$ can be considered to be a uniformly dense subspace of $B\left(R^{*}\right)$, the totality of bounded real-valued continuons functions on $R^{*}$. We call the set

$$
\Gamma=R^{*}-R
$$

the Royden boundary of $R$ (see Nakai [5], [6]).

We denote by $\Delta$ the set of all regular points in $\Gamma$ with respect to the Dirichlet problem considered for harmonic functions on $R$ with boundary values on $\Gamma$. This set $\Delta$ coincides with the harmonic boundary of $R$ named by Royden [9], i.e.

$$
\Delta=\left(p \in \Gamma ; f(p)=0 \text { for all } f \text { in } M_{\Delta}(R)\right)
$$


where we denote by $M_{\Delta}(R)$ the $B D$-closure of $M_{0}(R)$, the totality of functions in $M(R)$ with compact carriers in $R$. Here a sequence $\left(f_{n}\right)$ in $M(R)$ converges to $f$ in $M(R)$ in $B D$-topology, if, by definition, $\left(f_{n}\right)$ is uniformly bounded and converges to $f$ uniformly on each compact subset on $R$ and $D_{R}\left(f_{n}-f\right) \rightarrow 0$ as $n \rightarrow \infty$ (see Nakai [5], [6], [8]). We must notice that $g(z, o)$ is continuous on $R^{*}$ and vanishes on $\Delta$, since $\min (g(z, o),-\log \varepsilon)$ belongs to $M_{\Delta}(R)$.

We denote by $\mu$ the canonical measure on $\Gamma$ with center $o \in R$, which is defined as the regular Borel measure on $\Gamma$ satisfying

$$
u(o)=\int_{\Gamma} u(p) d \mu(p) \text { for any } u \text { in } H D(R),
$$

where $H D=H D(R)$ is, as usual, the totality of harmonic functions on $R$ with finite Dirichlet integrals taken over $R$. We know that the support $S_{\mu}$ of $\mu$ is identical with $\Delta$ (see Nakai [6]). Let $X$ be a Borel subset of $\Gamma$ and $f_{X}$ be the characteristic function of $X$ in $\Gamma$. We know that $f_{X}$ is resoltive and the generalized solution of the Dirichlet problem with boundary value $f_{X}$, denoted by $H^{f x}(z)$, is related to the canonical measure $\mu$ by

$$
H^{f_{X}}(o)=\mu(X) .
$$

Hence $\mu$ is the so-called harmonic measure and so the set $T-\Delta$ is of harmonic measure zero (see Nakai [8]).

5. For each Green line $L_{\theta}$ in $\$$, we set

$$
e_{\theta}=\bar{L}_{\theta}-L_{\theta}-(o),
$$

where $\bar{L}_{\theta}$ is the closure of $L_{\theta}$ in $R^{*}$. We call $e_{\theta}$ the end part of $L_{\theta}$. We also denote

$$
d_{\theta}=\sup \left(r(z) ; z \in L_{\theta}\right) .
$$

Clearly $\varepsilon<d_{\theta} \leq 1$. If $d_{\theta}<1$, then we call $L_{\theta}$ a singular Green line. We denote

$$
\mathbf{N}=\left(\theta \in J ; d_{9}<1\right) .
$$

If $\theta \in \mathbf{E}$, then $e_{\theta}$ is one point $z$ in $R$ at which $d \theta(z)=0$ and so we get $\mathbf{E} \subset \mathbf{N}$.

For any set $S$ in $I$, we denote by $\widetilde{S}$ and $\breve{S}$ the sets in $\mathbf{J}=\partial(5$ defined by

$$
\widetilde{S}=\left(\theta \in \mathbf{J} ; e_{\theta} \cap S \neq \varnothing\right)
$$

and 


$$
\check{S}=\left(\theta \in \mathbf{J} ; e_{\theta} \subset S\right) .
$$

Clearly $\widetilde{S} \supset \breve{S}$. The following is one of the fundamental lemmas of our discussion.

LEMMA 3. Let $K$ be a compact set in $I-\Delta$. Then $m(\widetilde{K})=0$.

Proof. We take an open neighborhood $U$ of $K$ in $R^{*}$ such that the relative boundary $\partial(U \cap R)$ of $U \cap R$ consists of a countable number of piece-wise analytic Jordan curves not accumulating in $R$ and $U_{n} \Delta=\phi$. We set

$$
U_{n}=U-\bar{R}_{n},
$$

where $\left(R_{n}\right)_{1}^{\infty}$ is a normal exhaustion of $R$. Then there exists a unique continuous function $w_{n}$ in $M(R)$ such that

$$
w_{n}= \begin{cases}1, & \text { on } U_{n} ; \\ \text { harmonic, } & \text { in } R-U_{n} ; \\ 0, & \text { on } \Delta .\end{cases}
$$

Then it holds that $w_{n} \searrow 0$ and $D\left(w_{n}\right) \searrow 0$ as $n \nearrow \infty$ (see p. 161 in Nakai [8]). Next we set

$$
U_{n}^{\prime}=\left(\theta ; L_{\theta} \in \mathbb{S}, L_{\theta} \cap\left(U_{n} \cap R\right) \neq \varnothing\right) .
$$

Clearly $U_{n}^{\prime}$ is open in $\mathbf{J}$ and $U_{n}^{\prime} \supset U_{n+1}^{\prime} \supset \tilde{K}$. For each $\theta$ in $U_{n}^{\prime}$, we choose a point $z_{\theta}$ in $L_{\theta} \cap\left(U_{n} \cap R\right)$. Then $w_{n}\left(z_{\theta}\right)=1$ and so

$$
1-w_{n}\left(\varepsilon e^{i \theta}\right)=\int_{\varepsilon}^{r\left(z_{\theta}\right)} \frac{\partial}{\partial r} w_{n}\left(r e^{i \theta}\right) d r .
$$

We can ind a positive number $a$ such that $1-w_{n}(z)>a>0$ on $\mathbf{J}$ for all $n$. Then

$$
a<\int_{\varepsilon}^{r\left(z_{\theta}\right)} \frac{\partial}{\partial r} w_{n}\left(r e^{i_{\theta}}\right) d r
$$

By Schwarz's inequality,

$$
\begin{aligned}
a^{2} & <\int_{\varepsilon}^{r\left(z_{\theta}\right)}\left|\frac{\partial}{\partial r} w_{n}\left(r e^{i_{\theta}}\right)\right|^{2} r d r \int_{\varepsilon}^{r\left(z_{\theta}\right)} \frac{d r}{r} \\
& \leq(-\log \varepsilon) \cdot \int_{\varepsilon}^{d_{\theta}}\left|\frac{\partial}{\partial r} w_{n}\left(r e^{i \theta}\right)\right|^{2} r d r .
\end{aligned}
$$

Hence we have 


$$
\begin{aligned}
\int_{U^{\prime} n} a^{2} d \theta & \leq(-\log \varepsilon) \int_{U_{n}^{\prime}} \int_{\varepsilon}^{d_{\theta}}\left(\left|\frac{\partial}{\partial r} w_{n}\left(r e^{i_{\theta}}\right)\right|^{2}+r^{-2}\left|\frac{\partial}{\partial \theta} w_{n}\left(r e^{i_{\theta}}\right)\right|^{2}\right) r d r d \theta \\
& \leq(-\log \varepsilon) D_{k}\left(w_{n}\right)
\end{aligned}
$$

Therefore by putting $c=(-\log \varepsilon) / 2 \pi a^{2}$, we get

$$
m\left(U_{n}^{\prime}\right) \leq c D_{R}\left(w_{n}\right) .
$$

Thus by noticing $U_{n}^{\prime} \supset U_{n+1}^{\prime} \supset \widetilde{K}$, we get

(outer Lebesgue measure of $\widetilde{K}) \leq \lim _{n} m\left(U_{n}^{\prime}\right) \leq \lim _{n} c D\left(W_{n}\right)=0$.

Hence $m(\widetilde{K})=0$.

Q.E.D.

As a corollary of our Lemma 3, we get the following well-known result due to Brelot-Choquet [2], which is a fundamental result in the theory of Green lines.

Lemma 4 (Brelot-Choquet's lemma). $m(\mathrm{~N})=0$, i.e. $d_{\theta}=1$ almost everywhere on $\mathbf{J}$.

Proof. Let $K_{n}=(p \in \Gamma ; g(p, o) \geq 1 / n)(n=1,2, \ldots)$. Then each $K_{n}$ is com. pact in $\Gamma-\Delta$ and

$$
\mathbf{N} \subset \mathbf{E} \cup\left(\widetilde{\cup_{n=1}^{\infty} K_{n}}\right)
$$

Since $\mathbf{E}$ is countable, $m(\mathbf{E})=0$. By Lemma 4 , since $K_{n}$ is compact in $I^{\prime}-$ $\Delta, m\left(\hat{K}_{n}\right)=0$ and so $m\left(\widetilde{\cup_{n=1}^{\infty} K_{n}}\right)=m\left(\cup_{n=1}^{\infty} \widetilde{K}_{n}\right)=0$. Hence $m(\mathrm{~N})=0$. Q.E.D.

\section{Functions with radial limits}

6. We say that a complex-valued function $f$ on $R$ possesses a radial limit almost everywhere on $\mathbf{J}$ if $\lim _{r \rightarrow 1} f\left(r e^{i \theta}\right)$ exists for any $\theta$ in $\mathbf{J}-\mathbf{N}$ except a set of Lebesgue measure zero. Here the meaning of the above limit is as follows:

$$
\lim _{r \rightarrow 1} f\left(r e^{i \theta}\right)=\lim _{r(z)>1, z \in L_{\theta}} f(z) .
$$

We denote the totality of complex-valued functions on $R$ possessing a radial limit almost everywhere on $\mathbf{J}$ by the notation $\mathfrak{R}=\mathfrak{N}(R)$.

We also denote by $F(R)$ the vector space of all real-valued a.c.T. functions on $R$ with finite Dirichlet integrals taken over $R$ (see Nakai [6]). Clearly $F(R) \supset H D(R)$ and $F(R) \supset M(R) \supset H B D(R)=H D(R) \cap H B(R)$. Although the following result follows from the result of Godefroid [3], we shall give an alternating proof. 
Proposition 2. $F(R) \subset \Re(R)$.

Proof. Let $L^{2}(\mathbf{J}, d m)$ be the Hilbert space of all square integrable functions on $\mathbf{J}$ with respect to the measure $m$ and $\|\cdot\|$ be the norm in $L^{2}(\mathbf{J}, d m)$. Let $f$ be in $F(R)$ and set $f_{r}(\theta)=f\left(r e^{i \theta}\right)$. Then $\left(f_{r}\right)_{0<r<1}$ can be considered to be a one-parameter family of functions in $L^{2}(\mathbf{J}-\mathbf{N}, d m)=L^{2}(\mathbf{J}, d m)$. For any $\theta$ in $\mathbf{J}-\mathbf{N}$ except a set of measure zero, by the definition of a.c.T. functions, we get

$$
f_{b}(\theta)-f_{a}(\theta)=\int_{a}^{b} \frac{\partial}{\partial r} f\left(r e^{i \theta}\right) d r \quad(0<a \leq b<1) .
$$

Hence by Schwarz's inequality,

$$
\begin{aligned}
\left|f_{b}(\theta)-f_{a}(\theta)\right|^{2} & \leq \int_{a}^{b}\left|\frac{\partial}{\partial r} f\left(r e^{i \theta}\right)\right|^{2} r d r \cdot \int_{a}^{b} \frac{d r}{r} \\
& \leq\left(\log \frac{b}{a}\right) \int_{a}^{b}\left|\frac{\partial}{\partial r} f\left(r e^{i \theta}\right)\right|^{2} r d r .
\end{aligned}
$$

Therefore

$$
\int_{0}^{2 \pi}\left|f_{b}(\theta)-f_{a}(\theta)\right|^{2} d \theta \leq\left(\log \frac{b}{a}\right) \int_{0}^{2 \pi} \int_{a}^{b}\left(\left|\frac{\partial}{\partial r} f\left(r e^{i \theta}\right)\right|^{2}+r^{-2}\left|\frac{\partial}{\partial \theta} f\left(r e^{i \theta}\right)\right|^{2}\right) r d r d \theta .
$$

Thus we get the following inequality:

$$
\left\|f_{b}-f_{a}\right\| \leq \sqrt{D_{G_{a, b}}(f)} \cdot \sqrt{\log \frac{b}{a}},
$$

where $G_{a, b}=G_{b}-\bar{G}_{a}(0<a<b<1)$.

Let $\left(K_{n}\right)_{1}^{\infty}$ be a sequence of compact sets in $\mathbf{J}-\mathbf{N}$ such that

$$
K_{n} \subset K_{n+1} \text { and } m\left(\cup_{n=1}^{\infty} K_{n}\right)=1 \text {. }
$$

For simplicity, we set $F=\cup_{n=1}^{\infty} K_{n}$. Let $\left(r_{n}\right)_{n=1}^{\infty}$ be a strictly increasing sequence of positive numbers such that $\lim _{n} r_{n}=1$ and $G_{r_{n}}$ is regular.

Since $f\left(r e^{i \theta}\right)$ is uniformly continuous on the compact set $\left(r e^{i \theta} ; r_{n} \leq r \leq r_{n+1}\right.$, $\left.\theta \in K_{n}\right)$, there exists a subdivision

$$
r_{n}=a_{n, 1}<a_{n, 2}<\cdots<a_{n, s(n)+1}=r_{n+1}
$$

of $\left[r_{n}, r_{n+1}\right]$ such that for any $r$ in $\left[a_{n, j}, a_{n, j+1}\right]$ and $\theta$ in $K_{n}$,

$$
\left|f\left(r e^{i \theta}\right)-f\left(a_{n, j} e^{i \theta}\right)\right|<1 / n,
$$

where $j$ is one of $1,2, \ldots$ and $s(n)$. Let $k$ be an arbitrary positive integer. 
Then $k$ is uniquely represented as

$$
k=\sum_{j=0}^{n-1} s(j)+t \quad(0 \leq t<s(n)),
$$

where we promise that $s(0)=0$. Using this expression, we define a new sequence $\left(b_{k}\right)_{k=1}^{\infty}$ by $b_{k}=a_{n, t}$.

Now set

$$
h(\theta)=\sum_{k=1}^{\infty}\left|f_{b_{k}}(\theta)-f_{b_{k+1}}(\theta)\right|
$$

for $\theta$ in $J-N$. By the triangle inequality,

$$
\begin{aligned}
\sqrt{\int_{0}^{2 \pi}|h(\theta)|^{2} d m(\theta)} & \leq \sum_{k=1}^{\infty}\left\|f_{b_{k}}-f_{b_{k+1}}\right\| \\
& \leq \sum_{k=1}^{\infty} \sqrt{D_{G_{b_{k}}, h_{k+1}}(f)} \cdot \sqrt{\log \frac{b_{k+1}}{b_{k}}} \\
& \leq \sum_{k=1}^{\infty}\left(D_{a_{b_{k}, b_{k+1}}}(f)+\log \frac{b_{k+1}}{b_{k}}\right) \\
& \leq D_{R}(f)+\log \frac{1}{b_{1}}
\end{aligned}
$$

Hence $h(\theta)<\infty$ almost everywhere on $\mathbf{J}$ and so on $F$. Hence

$$
\sum_{k=1}^{\infty}\left(f_{b_{k+1}}(\theta)-f_{b_{k}}(\theta)\right)
$$

converges almost everywhere on $F$. Since

$$
\sum_{k=1}^{\infty}\left(f_{b_{k+1}}(\theta)-f_{b_{k}}(\theta)\right)=\lim _{k} f_{b_{k}}(\theta)-f_{b_{1}}(\theta), f(\theta)=\lim _{k} f_{b_{k}}(\theta)
$$

exists almost everywhere on $F$. Let $F^{\prime}$ be the set of points in $F$ at which $f(\theta)$ exists. Then $m\left(F^{\prime}\right)=1$. Fix an arbitrary $\theta$ in $F^{\prime}$. Let $\eta$ be an arbitrary positive number. We can find a positive integer $n_{0}$ such that

$$
\theta \in K_{n_{0}} \text { and } 1 / n_{0}<\eta / 2 \text {. }
$$

Let $k_{0}$ be a positive integer such that $k_{0}>\sum \sum_{j=1}^{n_{0}} s(j)$ and that for any $k>k_{0}$

$$
\left|f_{b_{k}}(\theta)-f(\theta)\right|<\eta / 2 \text {. }
$$

Let $r$ be arbitrary in $b_{k_{0}}<r<1$. Then we can find a positive integer $k$ such that

$$
b_{k_{0}} \leq b_{k} \leq r<b_{k+1}
$$

Let the representation of $k$ by means of $(s(j))$ be

$$
k=\sum_{j=1}^{n} s(j)+t \quad(0 \leq t<s(n+1)) .
$$


Then clearly $n \geq n_{0}$ and $b_{k}=a_{n, t}$ and $\theta \in K_{n_{0}} \subset K_{n}$. Hence

$$
\left|f_{r}(\theta)-f_{b_{k}}(\theta)\right|=\left|f\left(r e^{i \theta}\right)-f\left(a_{n, t} e^{i \theta}\right)\right|<1 / n \leq 1 / n_{0}<\eta / 2 .
$$

Thus

$$
\left|f_{r}(\theta)-f(\theta)\right| \leq\left|f_{r}(\theta)-f_{b_{k}}(\theta)\right|+\left|f_{b_{k}}(\theta)-f(\theta)\right| \leq \eta / 2+\eta / 2=\eta .
$$

This shows that $\lim _{r \rightarrow 1} f_{r}(\theta)=f(\theta)$ exists for every $\theta$ in $F^{\prime}$.

Q.E.D.

7. For two numbers $a$ and $b$, we denote $a \cap b=\min (a, b)$ and $a \cup b=$ $\max (a, b)$. Similarly, for two harmonic functions $u$ and $v$, we denote by $u \wedge v$ the greatest harmonic minorant of $u$ and $v$ and by $u \vee v$ the least harmonic majorant of $u$ and $v$. We know that the class $H D(R)$ forms a vector lattice with respect to the lattice operations $\vee$ and $\wedge$ (see Nakai [6]). Concerning the general property of functions in $H D(R)$ on Green lines, we state the following.

Theorem 1.1 (Fatou type theorem). Any function $u$ in the class $H D(R)$ possesses the radial limit almost everywhere on $\mathrm{J}$, i.e.

$$
u(\theta)=\lim _{r \rightarrow 1} u\left(r e^{i \theta}\right)
$$

exists for every $\theta$ in $\mathbf{J}-\mathbf{N}$ except a set of Lebesgue measure zero.

Theorem 1.2 (Gauss type theorem). Let $u$ be in the class $H D(R)$. Then

$$
u(o)=\int_{0}^{2 \pi} u(\theta) d m(\theta) .
$$

TheOREM 1.3. For any pair of functions $u$ and $v$ in $H D(R)$, the function $u \wedge v$ belongs to $H D(R)$ and

$$
(u \wedge v)(\theta)=u(\theta) \cap v(\theta)
$$

for every $\theta$ in $\mathbf{J}-\mathrm{N}$ except a set of Lebesgue measure zero.

Proof of Theorem 1.1. Since $H D(R) \subset F(R)$, this follows from Proposition 2.

Proof of Theorem 1.3. As $H D(R)$ forms a vector lattice, so we may assume without loss of generality that $u$ and $v$ are non-negative. First we consider the case where $v$ is bounded. Let $f(z)=u(z) \cap v(z)$, which is a non-negative bounded superharmonic function on $R$ belonging to the algebra $M(R)$. We take a normal exhaustion $\left(R_{n}\right)_{1}^{\infty}$ of $R$ with $o \in R_{1}$. Let $\rho$ be in $0<\rho<1$ with regular 
$G_{\rho}$. We set $w_{\rho, n}$ the continuous functionon in $R$ such that

$$
w_{\rho, n}= \begin{cases}f, & \text { on } R-\overline{G_{\rho} \cap R_{n}}: \\ \text { harmonic, } & \text { on } G_{\rho} \cap R_{n} .\end{cases}
$$

Then it is easy to see that

$$
f \geq w_{\rho, n} \geq w_{\rho, n+1} \geq u \wedge v
$$

and

$$
w_{\rho^{\prime}, n} \leq w_{\rho, n} \quad\left(\rho^{\prime}>\rho\right)
$$

Thus we can define

$$
w_{\rho}=\lim _{n} w_{\rho, n}
$$

on $R$, which is harmonic in $G_{p}$ and continuous on $R$ and

$$
w_{p}(z)=u(z) \cap v(z)
$$

on $C_{p}$ and

$$
f \geq w_{\rho} \geq w_{\rho^{\prime}} \geq u \wedge v \quad\left(\rho^{\prime}>\rho\right) .
$$

Since $u \wedge v$ is the greatest harmonic minorant of $f$ and $\left(w_{p}\right)$ converges to a harmonic function on $R$ as $\rho \nearrow 1$, we can conclude that

$$
\lim _{p \rightarrow 1} w_{p}=u \wedge v
$$

on $R$. By Proposition 1,

$$
w_{\mathrm{P}}(0)=\int_{0}^{2 \pi} w_{\rho}\left(\rho e^{i \theta}\right) d m(\theta)=\int_{0}^{2 \pi} u\left(\rho e^{i \theta}\right) \cap v\left(\rho e^{i \theta}\right) d m(\theta) .
$$

Clearly $w_{\rho}(o) \searrow(u \wedge v)(o)(\rho \nearrow 1)$. On the other hand, by Theorem 1.1,

$$
u\left(\rho e^{i \theta}\right) \cap v\left(\rho e^{i \theta}\right) \rightarrow u(\theta) \cap v(\theta)
$$

as $\rho \rightarrow 1$ for every $\theta$ in $\mathbf{J}-\mathbf{N}$ except a set of Lebesgue zero, where $u(\theta)=$ $\lim _{\rho \lambda 1} u\left(\rho e^{i \theta}\right)$ and $v(\theta)=\lim _{\rho>1} v\left(\rho e^{i \theta}\right)$. Moreover, $u\left(\rho e^{i \theta}\right) \cap v\left(\rho e^{i \theta}\right)$ is uniformly bounded. Hence by Lebesgue's convergence theorem, we get, by making $\rho \nearrow 1$,

$$
(u \wedge v)(o)=\int_{0}^{2 \pi} u(\theta) \cap v(\theta) d m(\theta)
$$

On the other hand, by Proposition 1,

$$
(u \wedge v)(o)=\int_{0}^{2 \pi}(u \wedge v)\left(\rho e^{i \theta}\right) d m(\theta)
$$


and by Theorem 1.1, writing $(u \wedge v)(\theta)=\lim _{\rho \rightarrow 1}(u \wedge v)\left(\rho e^{i \theta}\right)$, we get

$$
(u \wedge v)(o)=\int_{0}^{2 \pi}(u \wedge v)(\theta) d m(\theta)
$$

Clearly $(u \wedge v)(\theta) \leq u(\theta) \cap v(\theta)$ almost everywhere on $\mathbf{J}-\mathbf{N}$ and

$$
\int_{0}^{2 \pi}(u(\theta) \cap v(\theta)-(u \wedge v)(\theta)) d m(\theta)=0 .
$$

Thus

$$
(u \wedge v)(\theta)=u(\theta) \cap v(\theta)
$$

almost everywhere on $\mathbf{J}-\mathbf{N}$.

Next we remove the assunption that $v$ is bounded. By the above consideration, we get

$$
\begin{aligned}
(u \wedge v)(\theta) \cap n & =((u \wedge v) \wedge n)(\theta)=(u \wedge(v \wedge n))(\theta)=u(\theta) \cap(v \wedge n)(\theta) \\
& =u(\theta) \cap(v(\theta) \cap n)=(u(\theta) \cap v(\theta)) \cap n,
\end{aligned}
$$

i.e.

$$
[(u \wedge v)(\theta)-u(\theta) \cap v(\theta)] \cap n=0
$$

almost everywhere on $\mathbf{J}-\mathbf{N}$ for any positive integer $n$. Thus by making n $\nearrow \infty$,

$$
(u \wedge v)(\theta)=u(\theta) \cap v(\theta)
$$

Proof of Theorem 1.2. Since $H D(R)$ forms a vector lattice, we may assume without loss of generality that $u \geq 0$. By Proposition 1 , we get

$$
(u \wedge n)(o)=\int_{0}^{2 \pi}(u \wedge n)\left(\rho e^{i \theta}\right) d m(\theta)
$$

for any positive integer $n$. Hence by Theorem 1.1, writing $(u \wedge n)(\theta)$ $=\lim _{\rho \rightarrow 1}(u \wedge n)\left(\rho e^{i \theta}\right)$, we get

$$
(u \wedge n)(o)=\int_{0}^{2 \pi}(u \wedge n)(\theta) d m(\theta)
$$

This with Theorem 1.3 gives

$$
(u \wedge n)(o)=\int_{0}^{2 \pi} u(\theta) \cap n d m(\theta)
$$

Clearly $(u \wedge n)(o) \nearrow u(o)$ and $u(\theta) \cap n \nearrow u(\theta)$ as $n \nearrow \infty$. Therefore by making 
$n \nearrow \infty$ we get

$$
u(o)=\int_{0}^{2 \pi} u(\theta) d m(\theta)
$$

8. We know that each function in $F(R)$ is continuously extended to $R^{*}$ admitting infinite values (see Nakai [6]). We denote

$$
F_{\Delta}^{\prime}(R)=(f \in F(R) ; f \text { vanishes on } \Delta) .
$$

Then $F_{\Delta}(R) \supset M_{\Delta}(R)$ and $f /(1+|f|) \in M_{\Delta}(R)$ for any $f$ in $F_{\Delta}(R)$ and the following harmonic decomposition holds (see Nakai [6]) :

$$
F(R)=H D(R) \oplus F_{\Delta}(R),
$$

i.e. any function $f$ in $F(R)$ is uniquely decomposed into the form $f=u+\varphi$ $\left(u \in H D(R), \varphi \in F_{\Delta}(R)\right)$, where we have

$$
D(u, \varphi)=0 \text { and } \sup _{R}|u|=\sup _{\Delta}|f| .
$$

Proposition 3. For any function $f$ in the class $F_{\Delta}(R)$, the radial limit $f(\theta)=\lim _{r \rightarrow 1} f\left(r e^{i \theta}\right)$, which exists almost everywhere on $\mathbf{J}-\mathbf{N}$ by Proposition 2, vanishes almost everywhere on $\mathrm{J}-\mathrm{N}$.

Proof. For each positive integer $n$, we set

$$
K_{n}=(p \in \Gamma ; f(p) \geq 1 / n) .
$$

Then $K_{n}$ is compact in $\Gamma-\Delta$ and so by Lemma $3, \boldsymbol{m}\left(\widetilde{K}_{n}\right)=0$. Next we set

$$
E_{n}=(\theta \in \mathbf{J} ; f(\theta) \geq 1 / n) .
$$

Clearly $E_{n}$ is measure equivalent to $\widetilde{K}_{n}$, i.e. $m\left(\widetilde{K}_{n} \ominus E_{n}\right)=0$, where $\widetilde{K}_{n} \ominus E_{n}=$ $\widetilde{K}_{n} \cup E_{n}-\widetilde{K}_{n} \cap E_{n}$. Thus $m\left(E_{n}\right)=0$.

As $(\theta \in \mathbf{J}-\mathbf{N} ; f(\theta)>0)=\cup{ }_{n=1}^{\infty} E_{n}$, so $m(\theta \in \mathbf{J}-\mathbf{N} ; f(\theta)>0)=0$.

Hence $f(\theta)=0$ almost everywhere on $\mathbf{J}-\mathbf{N}$.

Q.E.D.

We may consider $\mathbf{J}$ as a representation of the ideal boundary of $R$. Each function $f$ in $F(R)$ gives the "boundary function"

$$
f(\theta)=\lim _{r \rightarrow 1} f\left(r e^{i \theta}\right)
$$

on $\mathbf{J}$. By the harmonic decomposition of $f$,

$$
f(z)=u(z)+\varphi(z) \quad\left(u \in H D(R), \varphi \in F_{\Delta}(R)\right) .
$$

Hence by Proposition 3, 


$$
u(\theta)=\lim _{r \rightarrow 1} u\left(r e^{i \theta}\right)=\lim _{r \rightarrow 1}\left(f\left(r e^{i \theta}\right)-\varphi\left(r e^{i \theta}\right)\right)=f(\theta)
$$

almost everywhere on $\mathbf{J}$. Hence there exists a harmonic function $u$ with "boundary value" $f(\theta)$.

\section{Subsets of the space of Green lines}

9. We denote by $\bar{m}$ (resp. $\underline{m}$ ) the outer (resp. inner) measure on $\mathbf{J}$ induced by the normalized Lebesgue measure $m$.

Proposition 4.1. For any compact set $K$ in $\Gamma, \bar{m}(\widetilde{K}) \leq \mu(K)$.

Proposition 4.2. For any open set $U$ in $\Gamma, \underline{m}(\check{U}) \geq \mu(U)$.

These two propositions are equivalent. In fact, assume that Proposition 4.1 is true. If $U$ is open in $\Gamma$, then $I-U=K$ is compact in $\Gamma$ and

$$
\check{U}=(\mathbf{J}-\mathbf{E})-\tilde{K} .
$$

Hence we have

$$
\underline{m}(\breve{U})=1-\bar{m}(\widetilde{K}) \geq 1-\mu(K)=\mu(\Gamma-K)=\mu(U) .
$$

Conversely assume that Proposition 4.2 holds. If $K$ is compact in $\Gamma$, then $\Gamma-K=U$ is open in $\Gamma$ and

$$
\widetilde{K}=(\mathbf{J}-\mathbf{E})-\check{U}
$$

Therefore

$$
\bar{m}(\widetilde{K})=1-\underline{m}(\check{U}) \leq 1-\mu(U)=\mu(K) .
$$

Hence to prove these two propositions, it is sufficient to prove, for example, Proposition 4.2.

Proof of Proposition 4.2. Let $\eta$ be an arbitrary positive number. We set $F=\Gamma-U$. We can find a compact set $K$ in $\Delta \cap U$ such that

$$
\mu(K) \leq \mu(U)<\mu(K)+\eta \text {. }
$$

We can find a function $u$ in $H B D(R)$ such that

$$
0 \leq \boldsymbol{u} \leq 1
$$

on $R^{*}$ and

$$
u= \begin{cases}1, & \text { on } K ; \\ 0, & \text { on } \Delta-U\end{cases}
$$


(see Nakai [6]). We set

$$
F_{n}=(p \in F ; u(p) \geq 1 / n) .
$$

Clearly the set $F_{n}$ is compact and contained in $\Gamma-\Delta$ for any positive integer n. Then by Lemma 3 ,

$$
m\left(\widetilde{F}_{n}\right)=0
$$

Let

$$
F_{0}=F-\cup_{n=1}^{\infty} F_{n}=(p \in F ; u(p)=0) .
$$

Assume that $u(\theta)=\lim _{r \rightarrow 1} u\left(r e^{i \theta}\right)$ exists at $\theta$ in $\widetilde{F}_{0}$. Since $\tilde{L}_{\theta} \cap F_{0} \neq \varnothing, u(\theta)=0$. Hence by Proposition 2,

$$
\boldsymbol{u}(\theta)=0 \quad \text { almost everywhere on } \widetilde{F}_{0} .
$$

As $m\left(\widetilde{\cup}_{n=1}^{\infty} F_{n}\right)=m\left(\bigcup_{n=1}^{\infty} \widetilde{F}_{n}\right)=0$, so $m\left(\widetilde{F}-\widetilde{F}_{0}\right)=0$. Hence

$$
u(\theta)=0 \quad \text { almost everywhere on } \tilde{F} \text {. }
$$

Since $\Gamma=F \cup U$, we have

$$
\mathbf{J}-\mathbf{E}=\widetilde{F} \cup \check{U} .
$$

Set $V=(\theta ; u(\theta)>0)$. Then by the above

$$
m(V-\check{U})=0
$$

and $V$ is measurable. Hence

$$
\underline{m}(\breve{U}) \geq m(V) .
$$

On the other hand, by Proposition 1,

$$
\begin{aligned}
m(V) & =\int_{V} d m(\theta) \geq \int_{0}^{2 \pi} u(\theta) d m(\theta)=u(o) \\
& =\int_{\Gamma} u(p) d \mu(p) \geq \int_{K} d \mu(p)=\mu(K) .
\end{aligned}
$$

Hence

$$
\underline{m}(\check{U}) \geq \mu(U)-\eta
$$

and by making $\eta \searrow 0$, we get

$$
\underline{m}(\breve{U}) \geq \mu(U) .
$$

Remark 1. Proposition 4.1 implies that if $K$ is a compact set in $\Gamma$ with 
$\mu(K)=0$, then $\tilde{K}$ (and also $\check{K}$ ) is Lebesgue measurable and of measure zero.

Remark 2. By the increasing (resp. decreasing) monotone continuity of $\bar{m}$ (resp. $\underline{m}$ ) and the continuity of $\mu$, we may replace the compact set (resp. open set) in Proposition 4.1 (resp. 2) by $F_{\sigma}$ (resp. $G_{\delta}$ ) set.

10. As usual, we denote by $A D=A D(R)$ the class of all single valued analytic functions on $R$ with finite Dirichlet integrals taken over $R$.

Theorem 2.1 (Fatou type theorem). Any function $f$ in the class $A D(R)$ possesses a radial limit almost everywhere on $\mathrm{J}$, i.e.

$$
f(\theta)=\lim _{r \rightarrow 1} f\left(r e^{i \theta}\right)
$$

exists for every $\theta$ in $\mathrm{J}-\mathrm{N}$ except a set of Lebesgue measure zero.

Theorem 2.2 (F. and M. Riesz type theorem). Let $f$ belong to the class $A D(R)$ and $Z$ be a subset of $\mathbf{J}-\mathbf{N}$ with positive measure. Assume that

$$
f(\theta)=0
$$

for each $\theta$ in the set $Z$. Then $f$ vanishes identically on $R$.

Proof of Theorem 2.1. Since $\operatorname{Re}(f)$ and $\operatorname{Im}(f)$ belong to the class $H D(R)$, our assertion follows from Theorem 1.1.

Proof of Therem 2.2. We denote

$$
S=\cup_{\theta \in Z} e_{\theta} \quad \text { and } \quad K=\bar{S} .
$$

Clearly $K$ is a compact set in $\Gamma$ and

$$
\tilde{K} \supset \check{K} \supset \check{S}=Z .
$$

Hence by Proposition 4.1,

$$
\mu(K) \geq \bar{m}\{\tilde{K}) \geq m(Z)>0 .
$$

Since $\operatorname{Re}(f)$ and $\operatorname{Im}(f)$ are continuous on $R^{*}$ admitting infinite values, $f$ is continuous on $R^{*}$ admitting infinite values. As $f(\theta)=0$ for $\theta$ in $Z$, so $f$ must vanish on the set $e_{\theta}$ for each $\theta$ in $Z$. Thus $f$ vanishes on $S$ and by the continuity of $f, f$ vanishes on $K$. Thus the analytic function $f$ has continuous boundary value zero at each point of the compact set $K$ in $\Gamma$ with positive canonical measure. Hence by Lusin-Privaloff type theorem (see Nakai [8]), $f$ vanishes identically on $R$.

Q.E.D, 


\section{Measures concerning blocks}

11. Let $R \notin O_{H D}$. For each point $p$ in the harmonic boundary $\Delta$ of $R$, we set $\Lambda_{p}=(q \in \Gamma ; u(q)=u(p)$ for any $u$ in $H B D(R))$

and we call this set as the block at $p$. Since the class $H B D$ separates points in $\Delta$, we can conclude that

$$
\Lambda_{p} \cap \Delta=(p)
$$

and

$$
\Lambda_{p} \cap \Lambda_{q}=\varnothing \quad(p \neq q) .
$$

We can find a function $u$ in $H B D$ such that $u>0$ in $R$ and $u(p)=0$ (see Nakai [6]). Then $u=0$ on $A_{p}$. Multiplying $u$ by a suitable constant $a$, we get that $a u(z)>g(z, o)$ on $J$. Since $a u \geq g$ on 4 , we get $a u(z) \geq g(z . o)$ on $R^{*}-($. Thus

$$
g(q, o)=0 \text { on } A_{p}
$$

This shows that

$$
e_{\theta} \cap A_{p} \neq \varnothing \text { implies } d_{\theta}=1 \text { or } \theta \in \mathbf{J}-\mathbf{N} \text {. }
$$

Concerning blocks, we prove

Proposition 5. The set $\tilde{\Lambda}_{p}$ is measurable for any $p$ in $\Delta$ and

$$
m\left(\tilde{\Lambda}_{p}\right)=\mu(p) .
$$

Proof. First we consider the case where where $\mu(p)=0$. In this case, by Proposition 4.1, we have

$$
\bar{m}\left(\tilde{\Lambda}_{p}\right) \leq \mu(p)=0 .
$$

Hence $\tilde{\Lambda}_{p}$ is measurable and $\boldsymbol{m}\left(\tilde{\Lambda}_{p}\right)=\mu(p)$.

Next we assume that $\mu(p)>0$. Using the harmonic kernel $K(z, q)$ (see Nakai [6]), we set

$$
u(z)=\int_{(p)} K(z, q) d \mu(q) \quad(z \in R)
$$

Then $u(z)$ belongs to the class $\underline{H D}$ considered by Constantinescu-Cornea [1], where $\underline{H D}$ is the totality of limits of decreasing sequences of non-negative functions in the class $H D$. In particular, 


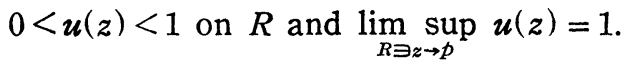

Hence we can find a decreasing sequence $\left(u_{n}\right)$ of functions $u_{n}$ in the class $H B D$ such that

$$
0<u_{n}(z)<1 \text { and } \lim _{n} u_{n}(z)=u(z)
$$

on $R$ (see Nakai [6]). Therefore $u_{n}(p)=1$ and so $u_{n}=1$ on $\Lambda_{p}$. For the sake of simplicity, we set

$$
a_{n}=u_{n}(o)-u(o) \geq 0 .
$$

For any $\rho$ in $0<\rho<1$ with regular $G_{\rho}$, we have, by Proposition 1 ,

$$
\int_{0}^{2 \pi}\left(u_{n}\left(\rho e^{i \theta}\right)-u\left(\rho e^{i \theta}\right)\right) d m(\theta)=a_{n} .
$$

Let

$$
\bar{u}(\theta)=\lim \sup _{\rho \rightarrow 1} u\left(\rho e^{i \theta}\right) .
$$

Since $u\left(\rho e^{i \theta}\right)$ is continuous in $\rho(0<\rho<1)$ and measurable in $\theta \in \mathbf{J}-\mathbf{N}, \bar{u}(\theta)$ is measurable on J. By Fatou's lemma,

$$
\begin{aligned}
& \int_{0}^{2 \pi} \lim \inf _{\rho \rightarrow 1}\left(u_{n}\left(\rho e^{i \theta}\right)-u\left(\rho e^{i \theta}\right)\right) d m(\theta) \\
& \leq \lim \inf _{\rho \rightarrow 1} \int_{0}^{2 \pi}\left(u_{n}\left(\rho e^{i \theta}\right)-u\left(\rho e^{i \theta}\right)\right) d m(\theta)=a_{n} .
\end{aligned}
$$

As we have

$$
\begin{aligned}
\lim \inf _{\rho \rightarrow 1}\left(u_{n}\left(\rho e^{i \theta}\right)-u\left(\rho e^{i \theta}\right)\right) & =u_{n}(\theta)-\lim \sup _{\rho \rightarrow 1} u\left(\rho e^{i \theta}\right) \\
& =u_{n}(\theta)-\bar{u}(\theta) \geq 0
\end{aligned}
$$

almost everywhere on $\mathbf{J}$, so we get

$$
0 \leq \int_{0}^{2 \pi}\left(u_{n}(\theta)-\bar{u}(\theta)\right) d m(\theta)=a_{n} .
$$

The sequence $\left(u_{n}(\theta)\right)_{n=1}^{\infty}$ is decreasing and so $v(\theta)=\lim _{n} u_{n}(\theta)$ exists and

$$
v(\theta) \geq \bar{u}(\theta)
$$

almost everywhere on $\mathbf{J}$. By making $n \nearrow \infty$, we get

$$
\int_{0}^{2 \pi}(v(\theta)-\bar{u}(\theta)) d m(\theta)=0 .
$$

Hence we get 


$$
v(\theta)=\bar{u}(\theta)
$$

almost everywhere on $\mathbf{J}$. Let

$$
U_{n, k}=\left(q \in \Gamma ; \boldsymbol{u}_{n}(q)>1-1 / k\right)
$$

and

$$
F_{n}=\left(q \in \Gamma ; u_{n}(q)=1\right) \text { and } F=\bigcap_{n=1}^{\infty} F_{n} .
$$

Then $F_{n}=\bigcap_{k=1}^{\infty} U_{n, k} \supset \Lambda_{p}$ and so $F \supset \Lambda_{p}$. By Propostion 2, there exists a set $J$ in $\mathbf{J}$ such that $\boldsymbol{m}\left(\mathbf{J}-J^{\prime}\right)=0$ and for any $\theta$ in $J^{\prime}, u_{n}(\theta)=\lim _{r \rightarrow 1} u_{n}\left(r e^{i \theta}\right)$ exists for all positive integers $n$. Then for any $n, u_{n}(\theta)=1\left(\theta \in \widetilde{F}_{n} \cap J^{\prime}\right)$ and so $u_{n}(\theta)$ $=1\left(\theta \in \widetilde{F} \cap J^{\prime}\right)$. Hence

$$
v(\theta)=1 \text { on } \widetilde{F} \cap J^{\prime} .
$$

Therefore, there exists a set $J^{\prime \prime}$ with $m\left(\mathbf{J}-J^{\prime \prime}\right)=0$ and

$$
\bar{u}(\theta)=1 \text { on } \tilde{F} \cap J^{\prime \prime} \text {. }
$$

Let $w$, be an arbitrary non-constant function in the class $\operatorname{HBD}(R)$. Let $c=\left(\sup _{z \in R}|w(z)-w(p)|\right)^{-1}$. Since $\lim \sup _{R \ni z \rightarrow p} u(z)=1$ and $\lim \sup _{R \ni z \rightarrow q} u(z)$ $=0(q \in \Delta ; q \neq p)$ (see Nakai $[6])$, we get

$$
\lim \inf _{R \ni z \rightarrow q}[(1-u(z))-c(w(z)-w(p))] \geq 0
$$

and

$$
\lim \inf _{R \ni z \rightarrow q}[(1-u(z))+c(w(z)-w(p))] \geq 0
$$

for any $q$ in $\Delta$. Hence by the maximum principle (see Nakai [6]), we get

$$
c|w(z)-w(p)| \leq 1-u(z)
$$

on $R$. Hence if $\theta \in \widetilde{F} \cap J^{\prime \prime}$, then we can find a sequence $r_{n} \nearrow 1$ such that

$$
\lim _{n} u\left(r_{n} e^{i \theta}\right)=\lim \sup _{r \rightarrow 1} u\left(r e^{i \theta}\right)=\bar{u}(\theta)=1 .
$$

Let $z_{n}=r_{n} e^{i \theta}$ and $q$ be an accumulation point of $\left(z_{n}\right)$. Then $q$ belongs to the set $e_{\theta}$ and as

$$
c\left|w\left(z_{n}\right)-w(p)\right| \leq 1-u\left(z_{n}\right),
$$

so we get $w(q)=w(p)$. This holds for any $w$ in $H B D(R)$, so $q \subseteq A_{p}$, or $e_{\theta} \cap A_{p}$ $\neq \varnothing$. Hence $\theta \in \tilde{\Lambda}_{p}$. Therefore

$$
\widetilde{F} \cap J^{\prime \prime} \subset \tilde{\Lambda}_{p} \subset \widetilde{F} .
$$


This shows that

$$
m\left(\tilde{F}-\tilde{\Lambda}_{p}\right)=0 .
$$

Then, since $\widetilde{F}_{n} \searrow \widetilde{F}$,

$$
\underline{m}\left(\tilde{\Lambda}_{p}\right)=\underline{m}(\widetilde{F})=\lim _{n} \underline{m}\left(\widetilde{F}_{n}\right) \text {. }
$$

By the fact that $\widetilde{U}_{n, k} \searrow \widetilde{F}_{n}$ and by Proposition 4.2 ,

$$
\begin{aligned}
\underline{m}\left(\widetilde{F}_{n}\right) & =\lim _{k} \underline{m}\left(\widetilde{U}_{n, k}\right) \geqq \lim _{k} \underline{m}\left(\check{U}_{n, k}\right) \geq \lim _{k} \mu\left(U_{n, k}\right) \\
& =\mu\left(F_{n}\right) \geq \mu\left(A_{p}\right)=\mu(p) .
\end{aligned}
$$

Hence

$$
\underline{m}\left(\tilde{\Lambda}_{p}\right) \geq \mu(p) \text {. }
$$

On the other hand, since $A_{p}$ is compact and $A_{p} \cap \Delta=(p)$, we get by using Proposition 4.1,

$$
\bar{m}\left(\tilde{\Lambda}_{p}\right) \leq \mu\left(\Lambda_{p}\right)=\mu(p) .
$$

Thus we get

$$
\mu(p) \leq \underline{m}\left(\tilde{\Lambda}_{p}\right) \leq \bar{m}\left(\tilde{\Lambda}_{p}\right) \leq \mu(p) .
$$

This shows that $\tilde{\Lambda}_{p}$ is measurable and $m\left(\tilde{\Lambda}_{p}\right)=\mu(p)$.

Q.E.D.

\section{Indivisible set in the space of Green lines}

12. Let $R$ be a hyperbolic Riemann surface and

$$
\underline{H D}=\underline{H D}(R)
$$

be the class of all functions on $R$ which are the limits of non-increasing sequences of non-negative functions in the class $H D(R)$. A function $u$ in the class $H D(R)$ is called an $\underline{H D}$-minimal function on $R$ if $u>0$ on $R$ and if for any $v$ in $\underline{H D}(R)$ with $u \geq v$ on $R$, there exists a constant $c_{v}$ with $v=c_{v} u$ on $R$. If $R$ carries at least one $H D$-minimal function, then, following Constantinescu-Cornea [1], we denote the fact by

$$
R \in U_{H D}
$$

In terms of the theory of Royden's compactification, this condition is characterized by the following (see Nakai [6]) :

$R \in U_{H D}$ if and only if there exists a point $p$ in $\Gamma$ with $\mu(p)>0$. 
Constantinescu-Cornea [1] gave the following characterization of the class $U_{H D}$ : let $R$ be hyperbolic. Then there exists an analytic mapping $\varphi$ of the unit disc $(z ;|z|<1)$ onto $R$. Consider the class $\mathfrak{S}$ of all functions $v$ on $(z ;|z|<1)$ such that $v=u \circ \varphi$ for some $u$ in $H D(R)$. Then it is proved that for any $v$ in $\mathscr{g}$

$$
\lim _{r \rightarrow 1} v\left(r e^{i \theta}\right)=v(\theta)
$$

exists almost everywhere on $C=(z ;|z|=1)$. Constantinescu-Cornea's characterization is as follows:

$R \in U_{H D}$ if and only if there exists a set $P$ of $C$ of positive measure such that $v(\theta)=\lim _{r \rightarrow 1} v\left(r e^{i \theta}\right)=$ const. almost everywhere on $P$ for any $v$ in $\mathscr{S}_{\text {. }}$

Here we give the similar result as above for the space of Green lines instead of the universal covering surface.

Theorem 3. In order that a hyperbolic Riemann surface $R$ should belong to the class $U_{H D}$, it is necessary and sufficient that there exists a measurable set $P$ in $\mathbf{J}-\mathbf{N}$ with $m(P)>0$ such that for any function $u$ in the class $H D(R), u(\theta)=$ $\lim _{r \rightarrow 1} u\left(r e^{i \theta}\right)$ is a constant almost everywhere on $P$.

Proof. First we show the necessity of our condition. Let $R \in U_{H D}$. Then there exists a point $p$ in $\Gamma$ with $\mu(p)>0$ (see Nakai [6]). Now we show that $P=\tilde{\Lambda}_{p}$ is the required set. ${ }^{*)}$ By Proposition 5,

$$
m(P)=m\left(\tilde{\Lambda}_{p}\right)=\mu(p)>0 .
$$

Next let $u \in H D(R)$. We must show that $u(\theta)$ is a constant almost everywhere on $P$. Since $H D(R)$ forms a vector lattice, we may assume that $u>0$ on $R$.

We denote by $u_{c}$ the harmonic function $u \wedge c$, where $c$ is a positive constant. Then by Theorem 1.3,

$$
u_{c}(\theta)=u(\theta) \cap c
$$

almost everywhere on $J$, where $u(\theta)=\lim _{r \rightarrow 1} u\left(r e^{i \theta}\right)$ and $u_{c}(\theta)=\lim _{r \rightarrow 1} u_{c}\left(r e^{i \theta}\right)$. We also have that

$$
u_{c}(q)=u(q) \cap c
$$

on $\Delta$ (see Nakai [6]). Since $\mu(p)>0, u(p)<\infty$. Hence for any $c>u(p)$,

$$
u_{c}(q)=u(p)
$$

\footnotetext{
*) If $R \in O_{H D}$, then our assertion is clear. So we assume that $R \notin O_{H D}$.
} 
for any point $q$ in the block $A_{p}$. Let $\left(c_{n}\right)_{1}^{\infty}$ be a sequence of numbers such that $u(p)<c_{n} \nearrow \infty$. Let $J^{\prime}$ be the subset of $\mathbf{J}$ with $m\left(\mathbf{J}-J^{\prime}\right)=0$ such that for any positive integer $n, u_{c_{n}}(\theta)$ exists for all $\theta$ in $J^{\prime}$. If $\theta \in P \cap J^{\prime}=\tilde{\Lambda}_{p} \cap J^{\prime}$, then $u_{c_{n}}$ is a constant on $e_{\theta}$ and $e_{\theta} \cap A_{p} \neq \varnothing$ and so $u_{c_{n}}$ is a constant $u(p)$ on the block $\Lambda_{p}$. Thus $\boldsymbol{u}_{c_{n}}(\theta)=\boldsymbol{u}(p)$. Hence for all positive integers $n$,

$$
u(\theta) \cap c_{n}=u_{c_{n}}(\theta)=u(p)
$$

and so

$$
u(\theta)=u(p)
$$

for any $\theta$ in $P \cap J^{\prime}$. Hence

$$
\lim _{r \uparrow 1} u\left(r e^{i \theta}\right)
$$

exists and is a constant for $\theta \in P \cap J^{\prime}$, where $m\left(P-P \cap J^{\prime}\right)=0$.

Next we show that our condition is sufficient. For the aim, we denote by $\widetilde{\mho}_{P}$ the set of all functions $u$ in $H D(R)$ such that

$$
0<u \leq 1
$$

on $R$ and

$$
u(\theta)=1
$$

almost cverywhere on $P$. For any $u$ and $v$ in $\mathfrak{F}_{P}$, by Theorem 1.3,

$$
(u \wedge v)(\theta)=u(\theta) \cap v(\theta)=1
$$

almost everywhere on $P$ and $0<u \wedge v \leq 1$ on $R$. Thus $u \wedge v$ belongs to $\mathfrak{F}_{P}$. Hence it is well-known that

$$
s(z)=\inf \left(u(z) ; u \in \mathfrak{F}_{P}\right) \quad(z \in R)
$$

is a harmonic function on $R$ and there exists a non-increasing sequence $\left(u_{n}\right)$ of functions in $\tilde{F}_{r}$ such that

$$
\lim _{n} u_{n}(z)=s(z)
$$

on $R$. Therefore $s(z)$ belongs to the class $H D(R)$. As we have

$$
s(o)=\lim _{n} u_{n}(o)=\lim _{n} \int_{0}^{2 \pi} u_{n}(\theta) d m(\theta) \geq \int_{P} d m(\theta)=m(P)>0,
$$

so we can conclude that 


$$
s(z)>0 \text { on } R \text {. }
$$

Now we show that $s(z)$ is $H D$-minimal on $R$. For the aim take an arbitrary function $t(z)$ in $\underline{H D}(R)$ such that $s(z) \geq t(z)>0$ on $R$. Let $\left(v_{n}^{\prime}\right)$ be the nonincreasing sequence of functions in the class $H D(R)$ such that

$$
\lim _{n} v_{n}^{\prime}(z)=t(z)
$$

Then, since the class $\underline{H D}$ is a vector lattice (see Nakai [6]), $v_{n}=u_{n} \wedge v_{n}^{\prime}$ belongs to the class $H D(R)$ and $v_{n} \searrow s \wedge t=t$. Hence

$$
\lim _{n} v_{n}(z)=t(z)
$$

on $R$ and

$$
1 \geq u_{n} \geq v_{n}>0
$$

on $R$. By the assumption on $P$,

$$
v_{n}(\theta)=c_{n} \quad(\text { a constant })
$$

almost everywhere on $P$. Clearly $0 \leq c_{n} \leq 1$ and there exists a constant $c$ in $0 \leq c \leq 1$ such that $c_{n} \downarrow c$.

If $c=1$, then $0<v_{n}<1$ and $v_{n}(\theta)=1$ almost everywhere on $P$. Hence $v_{n} \in$ $\tilde{\mho F}_{P}$ and so $v_{n} \geq s$ on $R$ or $t \geq s$. Hence $t=s$.

If $c<1$, then we may assume that $c_{n}<1$. Then

$$
\frac{u_{n}-v_{n}}{1-c_{n}} \text { and } \frac{u_{n}+v_{n}}{1+c_{n}} \wedge 1
$$

belong to the class $\mathfrak{\mho}_{P}$. Hence

$$
\frac{u_{n}-v_{n}}{1-c_{n}} \geq s \text { and } \frac{u_{n}+v_{n}}{1+c_{n}} \geq \frac{u_{n}+v_{n}}{1+c_{n}} \wedge 1 \geq s
$$

on $R$ and by making $n \nearrow \infty$, we get

$$
\frac{s-t}{1-c} \geq s \text { and } \frac{s+t}{1+c} \geq s
$$

on $R$. Then the first inequality shows that $s-t \geq s-c s$ or

$$
c s \geq t
$$

on $R$. Similarly, the second inequality gives that $s+t \geq s+c s$ or

$$
c s \leq t
$$


on $R$. Thus $t=c s$ on $R$.

Hence in any case, the function $t$ is a constant multiple of $s$ and so $s$ is $\underline{H D}$-minimal on $R$. Therefore we can conclude that $R \in U_{H D}$.

Q.E.D.

\section{References}

[1] C. Constantinescu and A. Cornea: Über den idealen Rand und einige seiner Anwendungen bei der Klassifikation der Riemannschen Flächen, Nagoya Math. J., 13 (1958), 169-233.

[2] M. Brelot et G. Choquet: Espaces et lignes de Green, Ann. Inst. Fourier, 3 (1951), 119-263.

[3] M. Godefroid: Une propriété des fonctions B.L.D. dans un espace de Green, Ann. Inst. Fourier, 9 (1959), 301-304.

[4] Y. Kusunoki and S. Mori: On the harmonic boundary of an open Riemann surface, Jap. J. Math., 29 (1960), 52-56.

[5] M. Nakai: On a ring isomorphism induced by quasiconformal mappings, Nagoya Math. J., 14 (1959), 201-221.

[6] M. Nakai: A measure on the harmonic boundary of a Riemann surface, Nagoya Math. J., 17 (1960), 181-218.

[7] M. Nakai: Bordered Riemann surface with parabolic double, Proc. Japan Acad., 37 (1961), 553-555.

[8] M. Nakai: Genus and classification of Riemann surfaces, Osaka Math. J., 14 (1962). $153-180$.

[9] H. L. Royden: The ideal boundary of an open Rimann surface, Ann. Math. Studies, 30 (1953), 107-109.

Mathematical Institute, Nagoya University 